\section{Chromosome positioning in interphase nuclei of hematopoietic stem cell and myeloid precursor}

\author{
Mariana Lomiento, Fabiana Mammoli, \\ Emilia Maria Cristina Mazza, \\ Silvio Bicciato, Sergio Ferrari \\ Department of Life Sciences, University \\ of Modena and Reggio Emilia, Modena, \\ Italy
}

\begin{abstract}
Human myelopoiesis is an intriguing biological process during which multipotent stem cells limit their differentiation potential generating precursors that evolve into terminally differentiated cells. The differentiation process is correlated with differential gene expression and changes in nuclear architecture. In interphase, chromosomes are distinct entities known as chromosome territories and they show a radial localization that could result in a constrain of inter-homologous distance. This element plays a role in genome stability and gene expression. Here, we provide the first experimental evidence of 3D chromosomal arrangement considering two steps of human normal myelopoiesis. Specifically, multicolor 3D-FISH and 3D image analysis revealed that, in both normal human hematopoietic stem cells and myelod precursors CD14-, chromosomal position is correlated with gene density. However, we observed that inter-homologue distances are totally different during differentiation. This could be associated with differential gene expression that we found comparing the two cell types. Our results disclose an unprecedented framework relevant for deciphering the genomic mechanisms at the base of normal human myelopoiesis.
\end{abstract}

\section{Introduction}

The organization of a eukaryotic nucleus reflects its specific expression profile, with dynamic rearrangements and repositioning involved in the regulation of gene expression and differentiation. Chromosomes represent the largest nuclear structural units and, in interphase, are organized in distinct entities known as chromosome territories (CTs) whose radial arrangement is nonrandom. ${ }^{1-3}$ In general CTs show a gene density driven positioning in spherical nuclei while a chromosome size driven positioning in ellipsoid nuclei. ${ }^{4}$ Moreover CTs radial localization preference could result in specific interchromosomal arrangment which are diverse in different cell types, ${ }^{3-7}$ during cell differentiation, development, ${ }^{8-10}$ and in cancer. ${ }^{11-13}$ The preferential spatial proximity of genomic loci likely enhances the probability of reciprocal chromosome rearrangement as chromosomal translocations, ${ }^{14,15}$ typical of pathologies such as Acute Myeloid Leukemia (AML).

On the other end it has been recently observed that homologous chromosomes are more distant than heterologous and a possible explanation is that the separation of homologous chromosomes could allow the maintenance of two copies of monoallelically expressed genes at a distance, avoiding their co-regulation, whereas other mechanisms would control transient interchromosomal contacts required for differential allelic expression. ${ }^{16}$

Given these evidences, the human myelopiesis, i.e., the process that leads to differentiation of myelopoietic cell lineages (erythroid, megakaryocytic, granulocytic and mono/macrophage), represents an ideal model to investigate chromosome positioning. Higher order chromatin organization during myelopoiesis has been recently studied considering specific chromatin features, ${ }^{17}$ while not chromosome position. Moreover several studies have demonstrated a differential gene expression during myeloid transition. ${ }^{18-20}$

In the present study, we used multicolor 3D-FISH and image analysis to reconstruct the chromosomal positioning during two steps of differentiation: normal human hematopoietic stem cells and derived myeloid precursors CD14-. Moreover we have measured the distance between the edges of homologs in the two cell types and we have observed a significant difference that can be associated with a differential gene expression, which occur during differentiation.

\section{Materials and Methods}

\section{Ethics statement}

Human CD34+ cells were purified upon donor's informed written consent from umbilical Cord Blood (CB) samples, collected after normal deliveries, according to the institutional guidelines for discarded material (Clearance of Ethical Commitee for Human Experimentation of Modena; Approval date: 18.01.2005; Approval file number \# 793/CE).
Correspondence: Mariana Lomiento, Department of Life Sciences, University of Modena and Reggio Emilia, Via Campi, 287 41100 Modena, Italy.

Tel.: +39.059.2055696 - Fax: +39.059.2055410. E-mail: mariana.lomiento@aosp.bo.it

Key words: Hematopoietic Stem Cell; Myelopoiesis; gene expression; 3D FISH; chromosome territory.

Acknowledgements: the authors thank Thomas Cremer and Mattia Forcato for useful discussions.

Contributions: ML and FM contributed equally. ML has performed the 3D experiments, analyzed the results and written the paper; FM has analyzed the images and the results; EMCM has performed the bioinformatic analysis; SB has supervised the bioinformatic analysis and analyzed the results; SF has supervised the whole project

Conflict of interest: the authors declare no potential conflict of interest.

Fundings: this work was supported by EuGESMA COST Action BM0801 (European Genomics and Epigenomics Study on MDS and AML) to S.F. and S.B. and from Epigenetics Flagship project to S.B.

Received for publication: 4 December 2017. Accepted for publication: 18 January 2018.

This work is licensed under a Creative Commons Attribution-NonCommercial 4.0 International License (CC BY-NC 4.0).

CCopyright M. Lomiento et al., 2018

Licensee PAGEPress, Italy

Hematology Reports 2018; 10:7515

doi:10.4081/hr.2018.7515

\section{CD34+ stem/progenitor and myeloid precursor CD14- cells purification}

Human CD34+ cells were purified from umbilical cord blood (CB) samples. Mononuclear cells were isolated by FicollHypaque (Lymphoprep; Nycomed Pharma, Oslo, Norway) gradient separation and washed twice with phosphate-buffered saline, and then CD34+ cells were separated using magnetic cell sorting procedure (EasySep Human CD34+ positive selection kit; StemCell Technologies, Vancouver, BC, Canada). CD34+ cell purity assessed by flow cytometry was $\geq 95 \%$ (Supplementary Figure S1). After immunomagnetic separation, CD34+ cells were seeded in 24-well plates at $5 \times 105 / \mathrm{mL}$ in Iscove's modified Dulbecco's medium (IMDM) (GIBCO, Grand Island, NY, USA) containing $20 \%$ Human Serum (Bio-Whittaker, Walkersville, MD, USA), SCF (50 ng/mL), Flt3-ligand 
(Flt3L) $(50 \mathrm{ng} / \mathrm{mL})$, TPO (20 ng/mL), IL-6 $(10 \mathrm{ng} / \mathrm{mL})$ and IL-3 (10 ng/mL) (all from R\&D Systems, Minneapolis, MN, USA) and fixed 24 hours later.

CD14- myeloid precursors were obtained by in vitro differentiation of $\mathrm{CB}$ derived CD34+ cells performed as already described. Briefly, CB CD34+ cells were cultured in IMDM added with $20 \%$ FCS (Bio-Whittaker, Walkersville, MD, USA), in the presence of human hematopoietic cytokines: SCF $(50 \mathrm{ng} / \mathrm{mL})$, Flt3-ligand (Flt3-1) $(50 \mathrm{ng} / \mathrm{mL})$, IL-6 $(10 \mathrm{ng} / \mathrm{mL})$ and IL$3(10 \mathrm{ng} / \mathrm{mL}$ ) (all from R\&D Systems, Minneapolis, MN, USA). After 7 days of culture, hematopoietic cells were analyzed, by flow cytometry, for CD14 antigen expression, estimated at about $15-20 \%$ of the entire cell population. Then CD14+ and CD14- cell fractions were obtained by immunomagnetic separation using a cell sorting procedure (EasySep ${ }^{\circledR}$ Human CD14 Positive Selection Kit, StemCell Technologies). ${ }^{21}$

\section{Cell fixation, and FISH pretreatment}

For 3D-FISH experiments, cells were allowed to attach to poly- L-lysinehydrobromide (Sigma) coated cover slips. Cells were fixed in $4 \%$ paraformaldehyde (PFA) in $0.3 \times$ PBS for $10 \mathrm{~min}$. Permeabilization steps included treatment with $0.5 \%$ Triton-X100 (15 min), with $20 \%$ glycerol in PBS for at least $1 \mathrm{~h}$, repeated freeze/thawing in liquid nitrogen, and incubation in $0.1 \mathrm{~N} \mathrm{HCl}$ (5 min) and pepsin solution $(2.5 \mathrm{mg} / \mathrm{mL}$ pepsin in $0.01 \mathrm{~N} \mathrm{HCL}$ at $37^{\circ} \mathrm{C}$ for $\left.5-10 \mathrm{~min}\right) .{ }^{10}$ Slides were stored at $4{ }^{\circ} \mathrm{C}$ in $50 \%$ formamide $/ 2 \times \mathrm{SSC}$. This technique is commonly used to preserve the 3D nuclear architecture and to study chromosome territory topology.

\section{Probe labeling, hybridization, and detection}

For 3D-FISH, the hybridization and detection protocol described by Solovei ${ }^{22}$ was followed. All DNA probes were labeled in the presence of Biotin-dUTP (Roche) or TexasRed- dUTP (Molecular Probes), respectively, and mixed with 10 -fold excess of human Cot-1 DNA. In situ hybridization was performed for $48 \mathrm{~h}$, followed by stringency washes for $3 \times 5 \mathrm{~min}$ in $0.1 \times \mathrm{SSC}$ $\left(60^{\circ} \mathrm{C}\right)$. Biotinylated probes were detected with Avidin-Alexa 488 (Molecular Probes). Metaphase chromosomes and 3D fixed interphase nuclei were counterstained for 10 min with DAPI $(2 \mu \mathrm{g} / \mathrm{mL})$.

\section{Digital microscopy and image processing}

Metaphase FISH images were captured with a Nikon Eclipse 90i system, equipped with Nomarski differential interference contrast (DIC) optics. Samples were photographed with a DS-5Mc Nikon digital camera and the resulting photographs were analyzed by using the Nikon AcU2 software program. Light optical serial sections of nuclei studied in three color 3D-FISH experiments were obtained with a laser scanning confocal microscope (LSM 410; Carl Zeiss MicroImaging) equipped with Ar and $\mathrm{He} / \mathrm{Ne}$ lasers. For imaging of multicolor 3D-FISH experiments a Leica TCS SP2 laser scanning confocal microscope (Leica Microsystems) with beam splitters tuned for DAPI, Alexa 488, and TexasRed was used. Nuclei were scanned with an axial distance of 200 $\mathrm{nm}$ between consecutive light optical sections yielding separate stacks of 8-bit gray-scale images for each fluorescence channel with a pixel size of $60 \mathrm{~nm}$. For each optical section, images were collected sequentially for all fluorochromes used, followed by correction of the axial chromatic shift for each fluorescence channel as described by Walter et al. ${ }^{23}$ Confocal image stacks were processed with ImageJ software (http://rsb.info.nih.gov/ij) using the deconvolution plugin to enhance resolution.

\section{Quantitative 3D data evaluation and} statistical analysis

Quantitative 3D evaluation of lightoptical serial sections was performed using voxel-based software algorithms eADS (enhanced Absolute 3D Distances to Surfaces). eADS software was used to determine the shortest distances (in $\mathrm{nm}$ ) between CT signals and the nuclear border and between the surface of the corresponding CT. The surface of the reference structure (CT, nucleus) was determined by applying a user set threshold: all voxels with intensities below the set threshold are set to zero. The surface of the reference structure contains all voxels with an intensity $>0$, which are directly adjacent to at least one voxel with an intensity equal to $0 .{ }^{16,24-26}$ For evaluations of distance measurements inside the nucleus deconvolution and normalization was

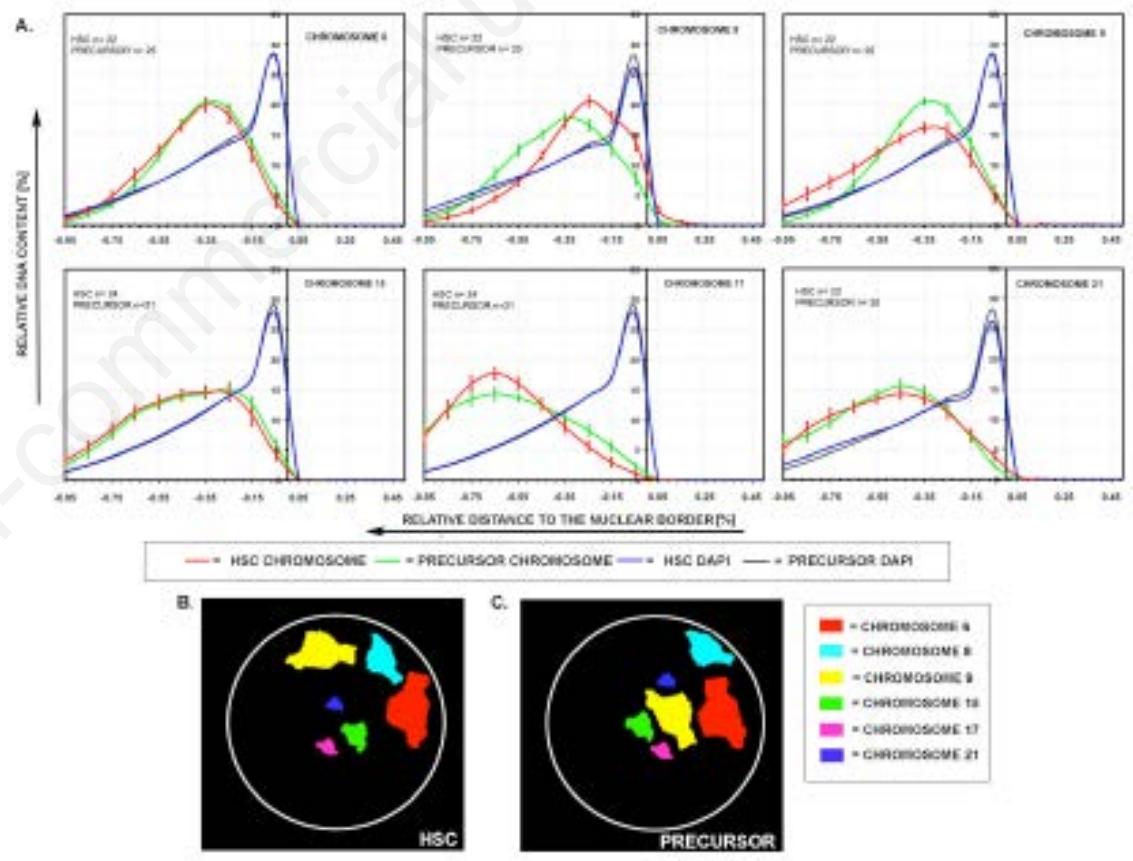

Figure 1. Quantitative 3D evaluation of the normalized localization of chromosome pairs $6,8,9,15,17$ and 21 in CD34+ HSCs and CD14- myeloid precursors (A.). The curves represent the quantitative evaluation of the radial probe distribution in the nucleus ( $n=$ number of nuclei). The $y$-axis reports the relative DNA content as the normalized sum of voxel intensities for the fluorochrome used for each CT, while in $\mathrm{x}$-axis is shown the normalized distance to the nuclear border. Negative values indicate the nuclear inside while " 0 " the nuclear border. The green curve represent the distribution of the chromosome in HSCs, the red curve the distribution of the chromosome in myeloid precursors, the blue curve indicates the HSCs nuclear counterstain and the grey curve the myeloid precursors counterstains. Reconstruction of the inferred map for the interphase localization of chromosomes 6, 8, 9, 15, 17, and 21 in hematopoietic stem cell (B.) and myeloid precursor nuclei (C.). Chromosome positions have been reconstructed from average distance values from the nuclear border. As such, homologous are thus shown as a single entity. 
performed to facilitate the comparison of different experiments. ${ }^{24}$ To test the data set for significant differences $(\mathrm{P}<0.05)$, the Mann-Whitney Rank Sum Test (U-Test) was applied on the results of the quantitative evaluation programs described above.

Chromosome territory volume (V), surface (S), and a roundness factor (RF) were computed. RF is defined as $R F=36$ $\rho \mathrm{V}^{2} / \mathrm{S}^{3}, 0<R F \leq I$.

We calculated nuclear radii $(\mathrm{Rn})$ and diameters $(\mathrm{Dn})$ from $\mathrm{R} n=(3 \mathrm{~V} / 4 \rho)^{1 / 3}$, where $\mathrm{V}$ is the thresholded nuclear pixel volume. We normalized the absolute spatial separations between chromosomes (CT1:CT2) as a fraction of nuclear diameter ((CT11:CT2)/Dn) to account for natural variations in nuclear size, which may influence relative positioning. ${ }^{15}$

Edge-to-edge distances were assessed using the absolute distance to surface (ADS) program. ${ }^{26,27}$ This program allowed for quantification of the shortest distance of given sub-CT units with respect to a boundary structure in $3 \mathrm{D}$. In these evaluations, overlapping homologues result in a null distance. The statistical significance of the inter-homologue and distances was tested using Student's t-test.

\section{Analysis of gene expression profiles}

Gene expression profiles of 12 hematopoietic stem cell (CD34+) and 11 progenitors (CD14-) samples were obtained from four different datasets downloaded from gene Expression Omnibus (Supplementary Table S1). Briefly, expression values were generated from Affymetrix HG-U133A raw intensity signals using the HG-U133A custom definition files for human GeneChips (version 15.0.0) based on Entrez gene (http://brainarray.mbni.med. umich.edu/Brainarray/Database/CustomCD F/15.0.0/entrezg.asp). Intensity values for a total of 12080 probe sets have been background adjusted, normalized using quantile normalization, and gene expression levels calculated using median polish summarization (Robust Multiarray Analysis, RMA). Differentially expressed chromosomal regions between CD34+ and CD14- samples have been identified using the LAP procedure, ${ }^{28}$ with the Significance Analysis of Microarray algorithm (SAM) two-class statistics coded in the PREDA R package. ${ }^{29}$ The percentage of false positive predictions was estimated with 10000 permutations and the q-value (i.e., False Discovery Rate, FDR) threshold was set to 0.5 . Functional over-representation analysis has been performed using DAVID (http://david.abcc.ncifcrf.gov/) and Gene Ontology Biological Process categories.

\section{Results and Discussion}

We first used multicolor 3D-FISH and 3D image analysis to localize chromosome positions with respect to the nuclear border in HSC CD34+ and myeloid precursor CD14- cells. Specifically, we investigated a total of six chromosomes, i.e., chromosome $6,8,9,15,17$ and 21, which are commonly implicated in translocations associated with acute myeloid leukemias, with the aim of evaluating if their nuclear position shows a preferential sub nuclear localization in each of the two cell populations. When considering the average chromosome position with respect to the nuclear border (Table 1) and the distribution of the chromosome territory (in term of relative DNA content with respect to the normalized distance to the nuclear border; Figure 1A), hematopoietic stem cells and myeloid precursors share the same localization for chromosomes $6,21,15$, and $17(\mathrm{P}>0.05)$ while the position of chromosomes 8 and 9 resulted significantly different $(\mathrm{P}=0.006$ and $\mathrm{P}=0.023$, respectively). Chromosome positions are consistent with average gene density, quantified as the ratio between the number of genes and the length in $\mathrm{Mb}$ of each chromosome (Table 1). In particular, chromosome 17 , which has the higher gene density (21.96 gene/Mb), has the more internal localization in both cell types ( $\mathrm{HSCs}=-1746.33 \mathrm{~nm}$; myeloid precursors $=$ $2312.43 \mathrm{~nm})$. On the contrary, chromosome 8 has the lower average gene density $(9.02$ gene $/ \mathrm{Mb}$ ) and is very peripheral in both cell types, although its position is closer to the nuclear border in precursors (Figure 1A). Interestingly, chromosome 9, characterized by an average gene density slightly higher than that of chromosome $8(10.80$ as compared to 9.02), results more internal in both the cell types, and particularly in myeloid precursors. Chromosome distances from the nuclear border allowed reconstructing a plausible map of the chromosome distribution during the differentiation of hematopoietic stem cells (Figure 1B) to myeloid precursors (Figure 1C). Homologous are shown as a single entity. Overall, positional data and the chromosomal positional map indicate that no drastic changes occur during differentiation of HSCs into CD14- myeloid precursors as regards to the chromosomal preferential localization within sub nuclear regions. This evidence is in perfect agreement with the relative small number of chromosomal regions found differentially expressed when comparing the gene expression profiles of CD34+ HSCs and CD14- myeloid precursors. Indeed, the regional analysis of transcriptional levels identified only 19 and 21 chromosomal regions up-regulated in HSCs and precursor cells, respectively (Supplementary Tables S2 and S3). Nevertheless, the functional annotation of differentially expressed regions found in this comparison supports a link between transcriptional differences and changes of chromatin organization during the transition from the stem to the precursor level, as recently highlighted. To investigate if indeed chromatin compaction changes during the transition from stem cells to precursors, we compared chromosome volumes, calculated from the images voxels, and found that the two cell types significantly differ for the

Table 1. Average chromosome position with respect to the nuclear border in hematopoietic stem cells (CD34+) and myeloid precursors (CD14-). P-values are calculated with a Mann-Whitney rank sum test (U-Test). The number of analyzed nuclei per chromosome per cell type was 30 .

\begin{tabular}{lccccc} 
Chromosome & $\begin{array}{c}\text { Average gene density } \\
\text { (gene/M/b) }\end{array}$ & $\begin{array}{c}\text { Average chromosome position (nm) } \\
\text { Precursors (CD14-) }\end{array}$ & P-value \\
6 & 12.01 & -1149.25 & -1266.07 & 0.098 \\
8 & 9.02 & -1154.85 & -794.01 & 0.006 \\
\hline 9 & 10.80 & -1174.93 & -1427.96 & 0.023 \\
15 & 12.35 & -1452.98 & -1899.18 & 0.261 \\
\hline 17 & 21.96 & -1746.33 & -2312.43 & 0.245 \\
21 & 9.35 & -1573.82 & -1345.54 & 0.4 \\
\hline
\end{tabular}


of gravity which is commonly use to study CT organization may not reflect interactions of these CT at their borders, for this kind of analysis we have performed an edge-to-edge evaluation.

We found that the inter-homologue distances were always shorter in precursor, significantly for chromosome 6, 9, and 15 (Figure 2 and Table 3).

Since the localization of the chromosomes is always consistent with gene density correlated distribution, the differences found can be most likely contacts could be required for differential allelic expression. ${ }^{8}$ As method of CT centers attributed to differential gene expression that can affect chromatin organization and compaction during the transition from the stem to the precursor level.

\section{Conclusions}

Multicolor 3D-FISH and 3D image analysis of chromosomal positioning during the differentiation of normal human hematopoietic stem cells into derived myeloid precursors indicate that there are no drastic changes in chromosomal preferential localization within sub nuclear regions during this differentiation transition. This evidence is in accordance with results obtained from the comparison of gene expression profiles of HSCs and myeloid precursor, where a relative small number of chromosomal regions were identified as differentially expressed. Nevertheless, the small differences in chromosomal radial positioning between the two cell types are accompanied by different chromosome volumes that could be correlated to variations of chromatin compaction occurring during differentiation. Moreover, stem cells and myeloid precursor present a high difference in homologous distances, which could be associated with differential gene expression.

Overall, these results represent the first experimental evidence of the arrangement of chromosomal position in two steps of normal human myelopoiesis and constitute a working framework relevant to decipher the genomic mechanisms at the base of myeloid differentiation.

\section{References}

1. Cremer T, Cremer M, Dietzel S, et al. Chromosome territories - a functional nuclear landscape. Curr Opin Cell Biol 2006;18:307-16.

2. Meaburn K, Misteli T. Chromosome territories. Nature 2007;445:379-81.

3. Cremer T, Cremer M. Chromosome territories. Cold Spring Harbor Perspect Biol 2010;2:a003889.

Table 3. Normalized average distance values between the homologs pair in each cell type. P-values are calculated with a Mann-Whitney rank sum test (U-Test). The number of analyzed nuclei per chromosome per cell type was 30 .

\begin{tabular}{lccc} 
Chromosome & $\begin{array}{c}\text { Average homologs distance }(\mathrm{nm}) \\
\text { Precursors (CD14-) }\end{array}$ & P-value \\
\hline 6 & 309,68 & 0.18 & $9.00 \mathrm{E}-004$ \\
8 & 318.25 & 267.2 & 0.09 \\
\hline 9 & 376.85 & 0.12 & $<0.0001$ \\
15 & 288.59 & 59.47 & $1.00 \mathrm{E}-004$ \\
\hline 17 & 129.01 & 111.96 & 0.19 \\
21 & 185.12 & 173.46 & 0.16 \\
\hline
\end{tabular}

4. Bolzer A, Kreth G, Solovei I, et al. Three-Dimensional Maps of All Chromosomes in Human Male Fibroblast Nuclei and Prometaphase Rosettes. PLoS Biol 2005;3:e157.

5. Mayer R, Brero A, von Hase J, et al. Common themes and cell type specific variations of higher order chromatin arrangements in the mouse. BMC Cell Biol 2005;6:44.

6. Parada L, McQueen P, Misteli T. Tissuespecific spatial organization of genomes. 
Genome Biol 2004;5:R44.

7. Zeitz M, Mukherjee L, Bhattacharya S, et al. A probabilistic model for the arrangement of a subset of human chromosome territories in WI38 Human fibroblasts. J Cell Physiol 2009;221:1209.

8. Kuroda M. Alteration of chromosome positioning during adipocyte differentiation. J Cell Sci 2004;117: 5897-903.

9. Stadler S, Schnapp V, Mayer R, et al. The architecture of chicken chromosome territories changes during differentiation BMC Cell Biol 2004;5:44.

10. Dixon J, Jung I, Selvaraj S, et al. Chromatin architecture reorganization during stem cell differentiation. Nature 2015;518:331-6.

11. Marella N, Bhattacharya S, Mukherjee L, et al. Cell type specific chromosome territory organization in the interphase nucleus of normal and cancer cells. $J$ Cell Physiol 2009;221:130-8.

12. Parada L, McQueen P, Munson P, Misteli T. Conservation of Relative Chromosome Positioning in Normal and Cancer Cells. Curr Biol 2002;12:16927.

13. Fritz A, Stojkovic B, Ding H, et al. Wide-scale alterations in interchromosomal organization in breast cancer cells: defining a network of interacting chromosomes. Hum Mol Genet 2014;23:5133-46.

14. Bickmore W, Teague P. Influences of chromosome size, gene density and nuclear position on the frequency of constitutional translocations in the human population. Chromosome Res
2002;10:707-15.

15. Roix J, McQueen P, Munson P, et al. Spatial proximity of translocation-prone gene loci in human lymphomas. Nat Genet 2003;34:287-91.

16. Heride C, Ricoul M, Kiêu K, et al. Distance between homologous chromosomes results from chromosome positioning constraints. J Cell Sci 2010;123:4063-75.

17. Hübner B, Lomiento M, Mammoli F, et al. Remodeling of nuclear landscapes during human myelopoietic cell differentiation maintains co-aligned active and inactive nuclear compartments. Epigenet Chromatin 2015;8.

18. Coppe A, Ferrari F, Bisognin A, et al. Motif discovery in promoters of genes co-localized and co-expressed during myeloid cells differentiation. Nucleic Acids Res 2008;37:533-49.

19. Ferrari F, Bortoluzzi S, Coppe A, et al. Genomic expression during human myelopoiesis. BMC Genomics 2007;8:264.

20. Tenedini E, Roncaglia E, Ferrari F, et al. Integrated analysis of microRNA and mRNA expression profiles in physiological myelopoiesis: role of hsamir-299-5p in CD34+ progenitor cells commitment. Cell Death Dis 2010;1(e28.

21. Montanari M, Gemelli C, Tenedini E, et al. Correlation between differentiation plasticity and mRNA expression profiling of CD34+-derived CD14- and CD14+ human normal myeloid precursors. Cell Death Different 2005;12:1588-600.
22. Solovei I, Cavallo A, Schermelleh L, et al. Spatial Preservation of Nuclear Chromatin Architecture during ThreeDimensional Fluorescence in Situ Hybridization (3D-FISH). Exp Cell Res 2002;276:10-23.

23. Walter J, Joffe B, Bolzer A, et al. Towards many colors in FISH on 3Dpreserved interphase nuclei. Cytogenet Genome Res 2006;114:367-78.

24. Küpper K, Kölbl A, Biener D, et al. Radial chromatin positioning is shaped by local gene density, not by gene expression. Chromosoma 2007;116:285306.

25. Lomiento M, Grasser F, Rocchi M, Müller S. The interplay between genome organization and nuclear architecture of primate evolutionary neo-centromeres. Genomics 2013;102:288-95.

26. Solovei I, Cavallo A, Schermelleh L, et al. Spatial Preservation of Nuclear Chromatin Architecture during ThreeDimensional Fluorescence in Situ Hybridization (3D-FISH). Exp Cell Res 2002;276:10-23.

27. Khalil A, Grant J, Caddle L, et al. Chromosome territories have a highly nonspherical morphology and nonrandom positioning. Chromosome Res 2007;15:899-916.

28. Callegaro A, Basso D, Bicciato S. A locally adaptive statistical procedure (LAP) to identify differentially expressed chromosomal regions. Bioinformatics 2006;22:2658-66.

29. Ferrari F, Solari A, Battaglia C, Bicciato S. PREDA: an R-package to identify regional variations in genomic data. Bioinformatics 2011;27:2446-7. 